\title{
Radiologic manifestations of acquired immunodeficiency syndrome
}

\author{
ABRAHAM PERA, D.O. \\ Lansing, Michigan \\ ROBERT A. WEISGRAU, M.D \\ THOMAS HARLE, M.D. \\ Houston, Texas \\ ADAN A. RIOS, M.D. \\ Missouri City, Texas
}

Acquired immunodeficiency syndrome, which was first identified in May 1981, has become one of the most important differentials in a large number of clinical presentations. The radiographic manifestations often are the first objective findings in these patients. The various radiographic presentations of this disease have been compiled into the three body systems most often affected. In the brain, the main involvement is direct infection by the HIV virus, unusual infections, and primary CNS lymphoma. The lungs most commonly are affected by Pneumocystis carinii pneumonia, along with other relatively unusual infections. The gastrointestinal tract is most frequently the host to Kaposi's sarcoma, lymphoma, and a myriad of rare infections. The imaging techniques not only identify the area of abnormalities, but they may also be used to direct biopsy, and for followup of these patients.

Acquired immunodeficiency syndrome (AIDS) is a severe compromise of cell-mediated immunity in a previously healthy individual. It is manifested clinically by life-threatening opportunistic infections and unusual malignancies. Up to this time, there have been more than 28,000 documented cases of AIDS in the United States, and it is estimated that there are more than 1 million individuals infected with the human immunodeficiency virus (HIV). Since its description in 1981, AIDS has been found mostly in homosexual men and intravenous drug abusers. However, heterosexual transmission also occurs; the present epidemic in Central Africa has spread mainly through heterosexual contacts. Infants born to infected mothers are also at high risk for contracting AIDS. Although recently emigrated Haitians were previously identified as a high-risk group, they have been dropped from this category by the Centers for Disease Control (CDC). The screening of donor blood for HIV virus has virtually eliminated the risk to hemophiliacs and blood transfusion recipients. ${ }^{1}$

While any organ system can be affected by this disease, the central nervous, gastrointestinal, and pulmonary systems are most often involved. The radiographic demonstration of certain abnormalities is often striking-but usually not specific. Multiple disease processes, infectious and neoplastic, often occur simultaneously because of the immunocompromised state.

The radiographic manifestations of AIDS in these three body systems are described in this paper. The importance of histologic and microbiologic correlation with radiographic findings is stressed as a guide to initiating appropriate and specific therapy.

\section{Central nervous system findings}

Patients with AIDS frequently have significant neurologic disorders. Psychologically, these patients have the symptoms of anxiety and depression that often are associated with other major debilitating illnesses. In the current social environment, they frequently are ostracized from the support groups that normally are available to patients with 


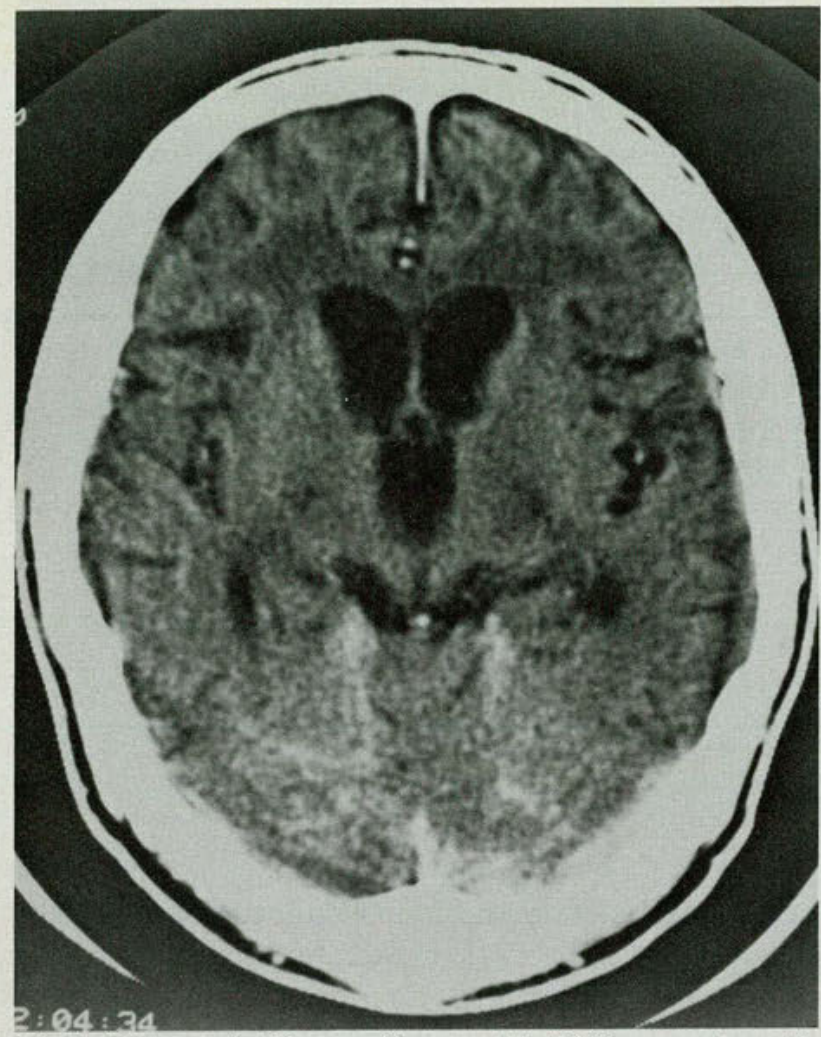

Fig. 1. CT scan of a 32-year-old man with AIDS presenting with nonfocal encephalopathy. The diffuse ventricular and sulcal enlargement is indicative of generalized atrophy. catastrophic illnesses. $^{2}$

Along with major psychiatric difficulties, neurologic symptoms are quite common. Nonfocal encephalopathy is observed in 50 percent of patients with AIDS at some time during their disease. Ten percent of patients have a neurologic manifestation as the initial presenting complaint. ${ }^{3}$ Clinically, these patients have subtle cognitive changes associated with mild depression, malaise, and withdrawal from social contact at onset of the disease. This progresses within weeks or months to incude more profound depression, psychomotor retardation, marked dementia, confusion, and incontinence. Seizure activity, hemi- or para-paresis, and coma leading to death can ensue. ${ }^{2}$ The cerebrospinal fluid (CSF) may demonstrate pleocytosis and increased protein and decreased glucose levels. These findings, however, are nonspecific. In one study, ${ }^{4}$ all patients with nonfocal encephalopathy demonstrated diffuse slowing on electroencephalography. Pathologically, there is moderate cerebral atrophy, areas suggestive of viral infection in gray and white matter of the brain and spinal cord, and white matter demyelination. ${ }^{4}$ The HIV virus has been identified in the brain cells of patients with AIDS ${ }^{5}$

Radiographically, the most frequent finding on computed tomography (CT) is diffuse atrophy, as evidenced by enlargement of the ventricles and sulci (Fig. 1). The focal abnormalities in the sub-

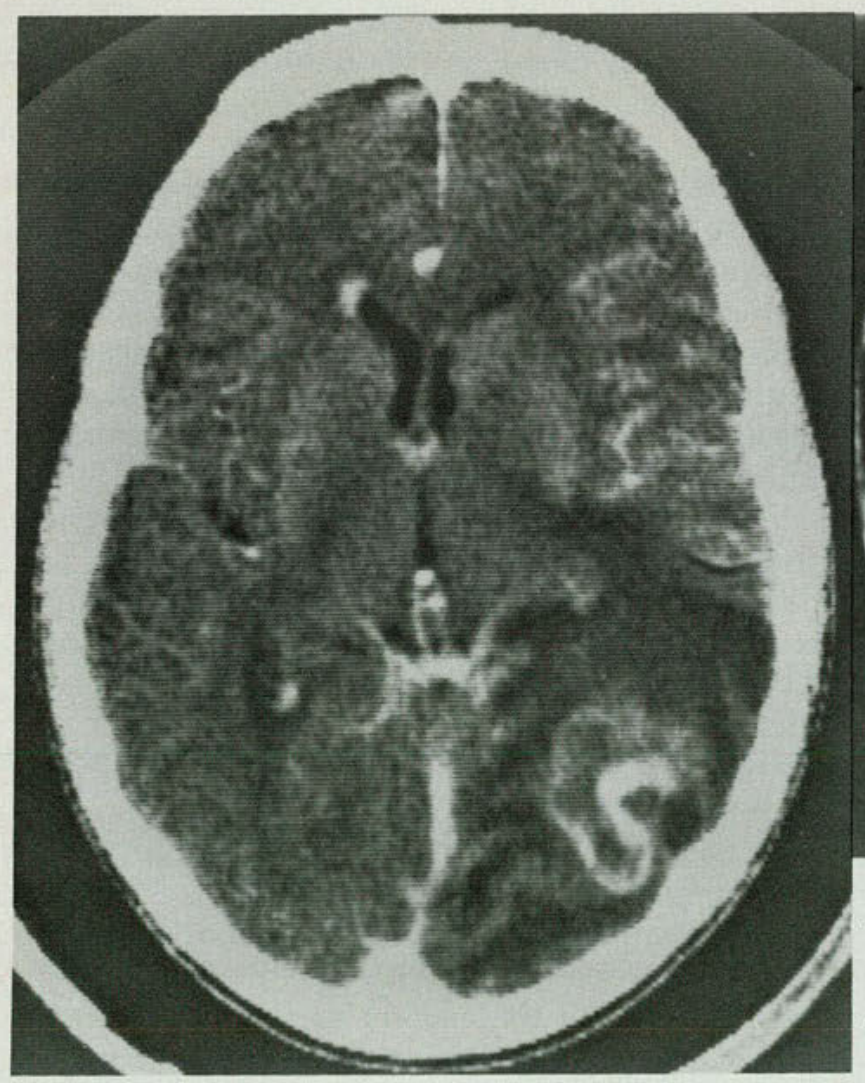

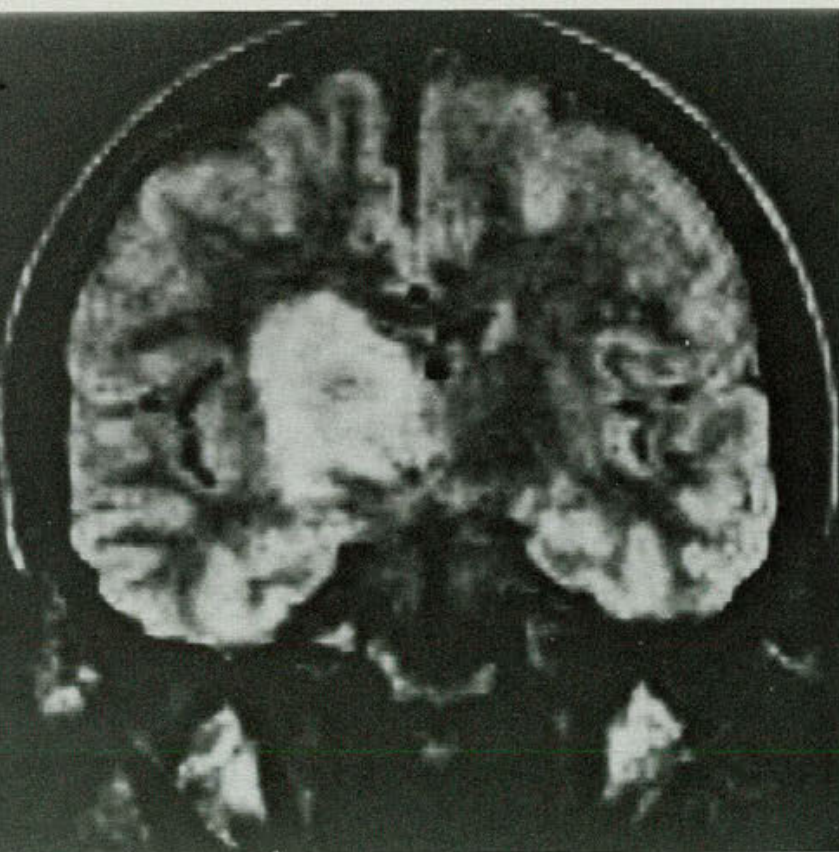

Fig. 2. CT scan of an AIDS patient with toxoplasmosis. There is gyral enhancement with surrounding edema and mass effect in the left occipital lobe; additional lesions are present in the right frontal horn region and the left basal ganglia. Fig. 3. Coronal MRI scan of patient with AIDS demonstrates toxoplasmosis involving the right thalamic region. 
acute cerebritis stage usually are not detectable. Small intensities in the white matter observed on magnetic resonance imaging (MRI) most likely demonstrate areas of demyelination and focal viral involvement. These intensities may represent vascular infarctions, other viral infections such as progressive multifocal leukoencephalopathy (PML), ${ }^{6}$ or even malignancies such as primary central nervous system (CNS) lymphoma. MRI is being recommended by some as the screening examination of choice in this setting. ${ }^{3}$ However, despite its high sensitivity, the procedure is nonspecific.

CNS infections are common and of varied etiology in AIDS patients. Toxoplasma gondii is found frequently (Figs. 2 and 3). The CT appearance of this protozoal infection ranges from focal edema to a mass with nodular enhancement, surrounding edema, and shift of midline structures. These lesions rarely are singular; usually, they are multiple and are scattered throughout the brain. In the patient with the proper clinical history, prompt empiric therapy has been advocated even prior to biopsy. ${ }^{6-8}$ Other infections of the CNS include tuberculosis (Fig. 4), atypical mycobacteria (most often Mycobacterium avium-intracellulare), and Candida; viral meningitis and meningitis secondary to Cryptococcus have also been described. ${ }^{6}$ Bacterial infections, embolic infarctions from terminal marantic endocarditis, and hemorrhage secondary to thrombocytopenia are other CNS complications of AIDS. ${ }^{7}$

The presence of primary CNS lymphoma is one of the major criteria used to identify an immunocompromised state as defined by the CDC. The radiographic appearance by $\mathrm{CT}$ and MRI of the CNS with lymphoma (Figs. 5-7) may be indistinguishable from that with infectious etiologies. Often, CNS lymphoma causes no symptoms during life, and its presence is demonstrated only on postmortem examination. ${ }^{4}$ Recently, Kaposi's sarcoma of the brain has also been reported, ${ }^{3}$ but this is a rare occurrence, and we have not identified a case at our institution.

It is of great importance to make a specific diagnosis of CNS disease to direct appropriate therapy. Except for positive cultures, the CSF findings are not unique to any one disease. Serum antibodies to specific agents may be positive because of previous infection, and even when active infection is present, rising antibody titers may not be noted because of the immunocompromised state. ${ }^{8}$ Biopsy is necessary for specific treatment of any CNS lesion identified by CT or MRI. Intraoperative ultrasoundguided biopsy can be of particular assistance in the treatment of small lesions located deep in the brain (Figs. 8 and 9).

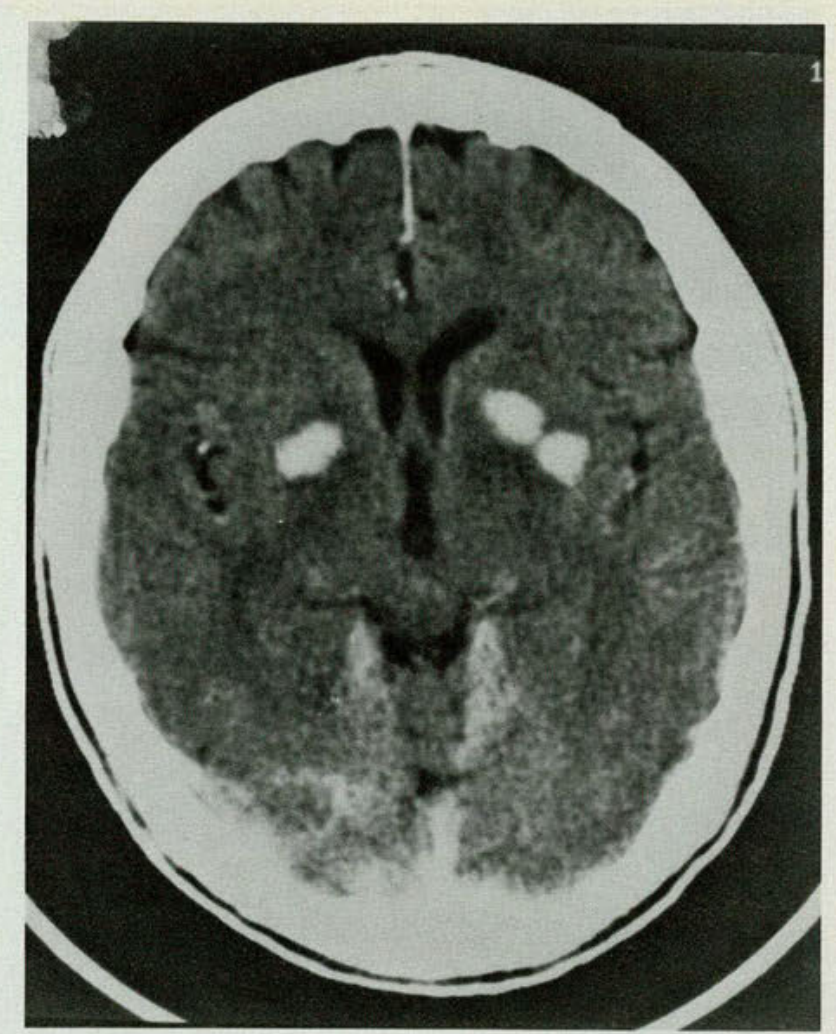

Fig. 4. CT scan of a 46-year-old male AIDS patient with multiple brain abscesses secondary to tuberculosis. There was subsequent improvement with antituberculous medication.

\section{Gastrointestinal complications}

Patients with AIDS are prone to primary and metastatic malignancy within the gastrointestinal tract and to infection by viral, protozoal, bacterial, and fungal organisms. In many patients, gastrointestinal complaints are the presenting symptomatology, or they soon become the predominant cause of long-term morbidity. Diagnosis of the specific entity causing the symptoms can prove difficult, because the etiology can be multifactorial. Often, specific treatment for an identified pathogen does not alter the symptomatology.

Gastrointestinal tract involvement can extend from the mouth to the anus. Xerostomia, odynophagia and dysphagia, cramping and bloating, diarrhea, and rectal pain are some of the more commonly encountered symptoms of gastrointestinal tract involvement in AIDS. Weight loss and malnutrition secondary to malabsorption are common. The radiologic identification of the specific pathologic process is hampered by a lack of specificity. Diagnosis of disease within the gastrointestinal tract, therefore, must combine identification of an abnormality by barium study, CT, ultrasonography, or a combination of imaging modalities with selective endoscopy, tissue biopsy, and microbiologic study. 

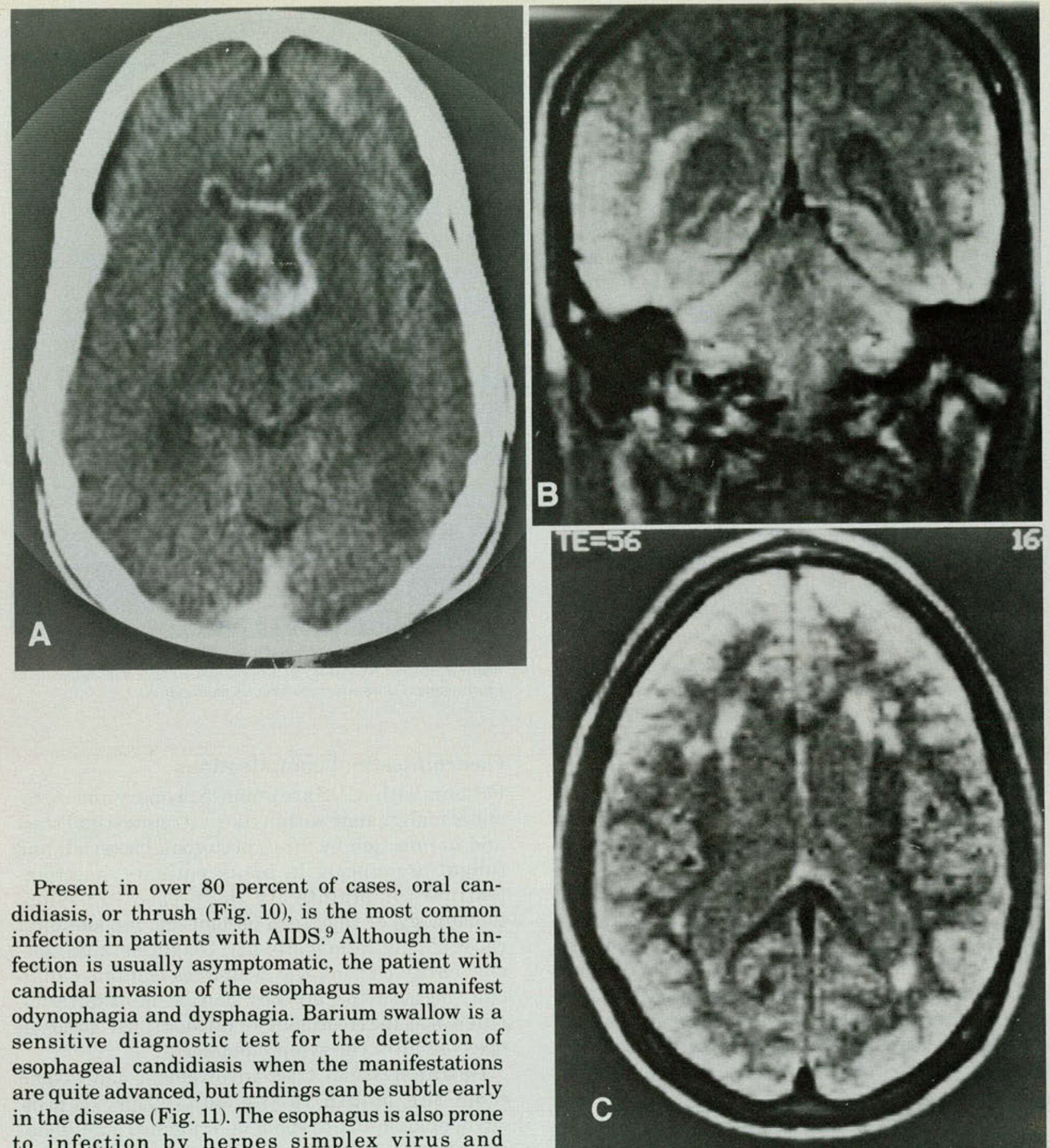

Present in over 80 percent of cases, oral candidiasis, or thrush (Fig. 10), is the most common infection in patients with AIDS. ${ }^{9}$ Although the infection is usually asymptomatic, the patient with candidal invasion of the esophagus may manifest odynophagia and dysphagia. Barium swallow is a sensitive diagnostic test for the detection of esophageal candidiasis when the manifestations are quite advanced, but findings can be subtle early in the disease (Fig. 11). The esophagus is also prone to infection by herpes simplex virus and cytomegalovirus, and the radiographic findings in these cases are difficult to distinguish from those of a patient with esophageal candidiasis. Although endoscopic biopsy is required for a precise diagnosis, the presence of oral candidiasis in a symp-

Fig. 5 (A). CT scan shows marked involvement of the periventricular region associated with primary CNS lymphoma in an AIDS patient. Figs. 6 (B) and 7 (C). Coronal and axial MRI scans of the brain demonstrate numerous areas of increased intensity secondary to CNS lymphoma, in this 32-year-old AIDS patient. 


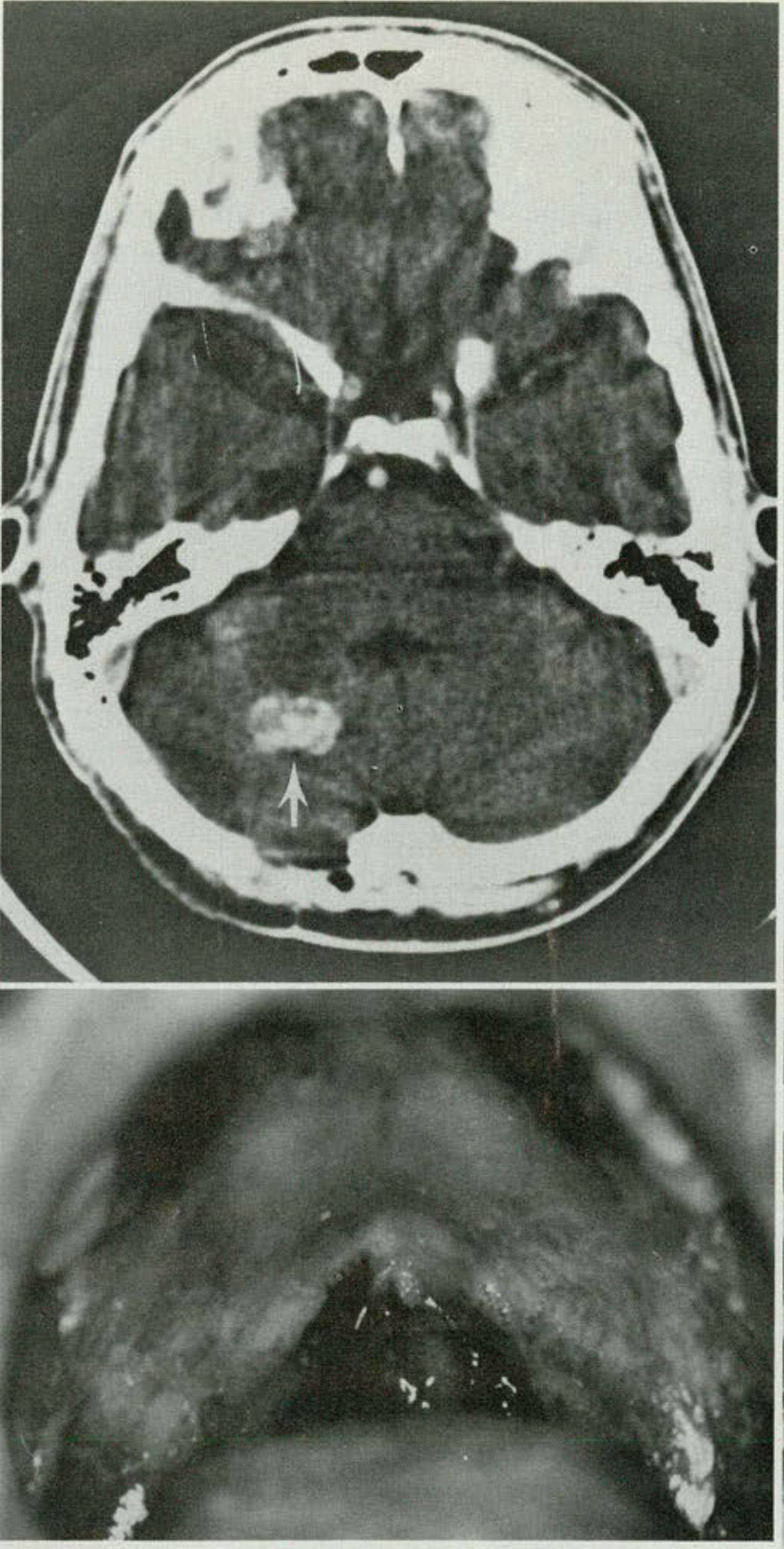

Fig. 10 (above). Typical appearance of thrush with white exudate in the oropharynx. Recurrent candidal infections are often seen in AIDS patients.

Fig. 11 (right). Barium swallow study shows candidal esophagitis with diffuse superficial ulcerations. This configuration, although striking, may also be seen with herpes simplex.

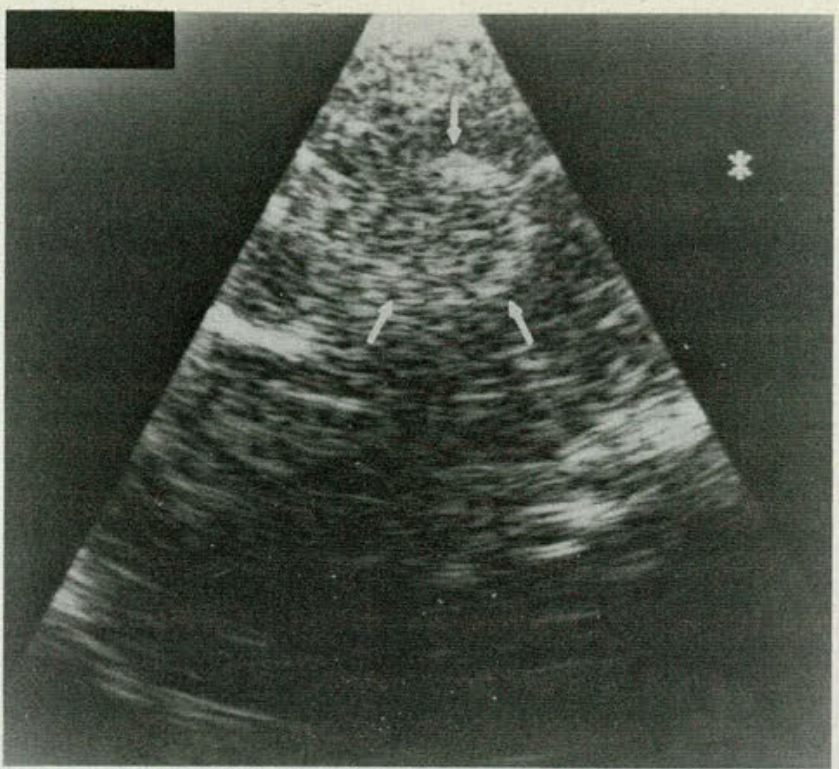

Figs. 8 (left) and 9 (right). Intraoperative ultrasound-guided biopsy proved this CT-detected cerebellar lesion from an AIDS patient to be lymphoma (arrows).

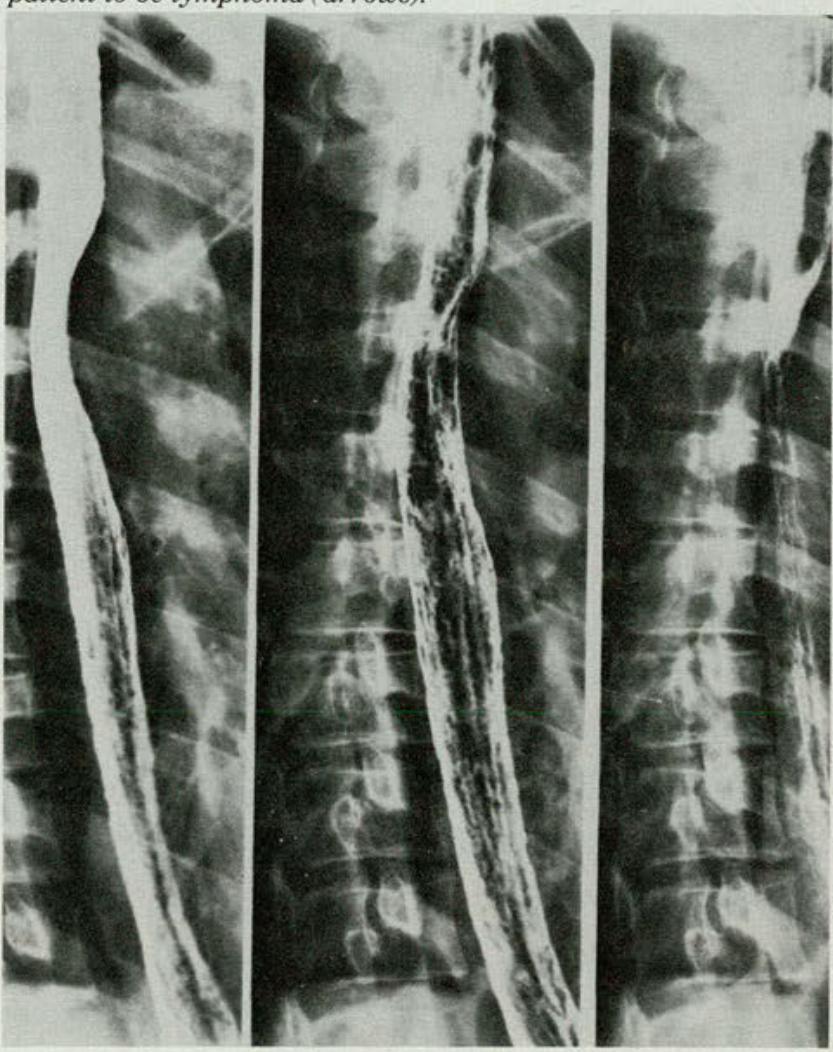




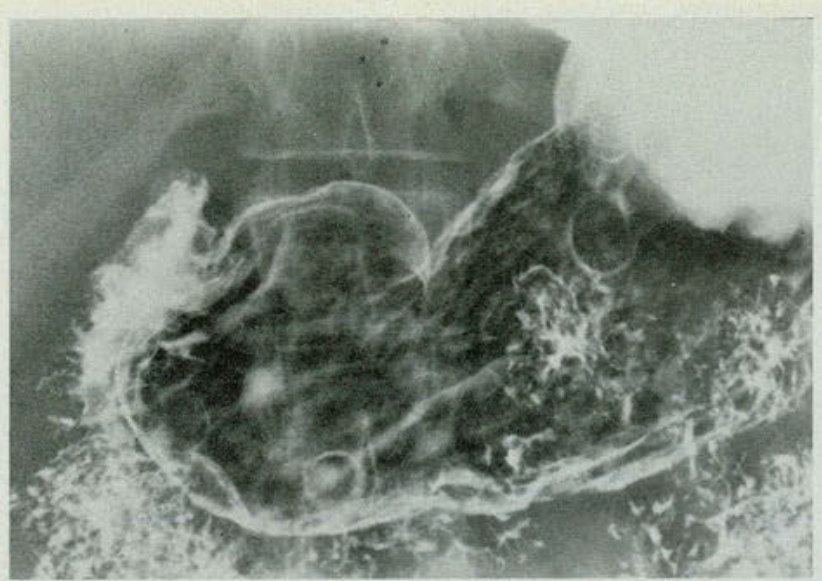

Fig. 12. Double-contrast barium examination of the stomach shows multiple target lesions typical of Kaposi's sarcoma.

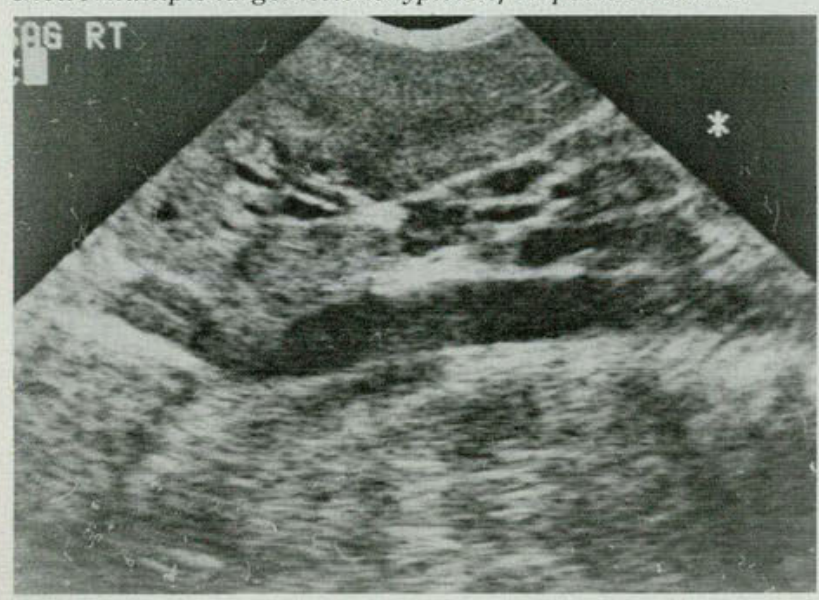

Fig. 13. Sagittal ultrasonographic scan of the midabdomen over the aorta demonstrates multiple enlarged lymph nodes around the pancreatic head; these are secondary to nonHodgkins lymphoma, in an AIDS patient. tomatic patient has been shown to be a sensitive predictor of the presence of esophageal involve ment by Candida. ${ }^{10}$

In patients with AIDS, the small bowel can be infected by viral, protozoal, bacterial, or mycobac terial organisms. In addition, it can be involved by malignancy. Patients may be asymptomatic, o they may suffer from abdominal pain, bloating cramping, profuse watery diarrhea, and weight los: secondary to malabsorption. Radiologic evaluation of any of these symptoms often includes an uppes gastrointestinal series and a small bowel barium study. The presence of submucosal lesions or poly poid defects (with or without central ulcerations located anywhere within the gastrointestinal trac should suggest the diagnosis of cancer (Fig. 12).

Kaposi's sarcoma was usually found in elderly men of Mediterranean ancestry. The low-grade malignancy usually involved the lower extremity anc rarely the gastrointestinal tract. In the AIDS population, Kaposi's sarcoma, a much more aggressive type, and lymphoma are the two most commonly associated gastrointestinal tract malignancies metastasis to other organs or involvement of lymph nodes (Fig. 13) is common. Diagnosis must rely or histologic tissue examination. When comparec with mucocutaneous Kaposi's sarcoma, the histology of disseminated Kaposi's sarcoma shows the lesions to have a more aggressive, angiosarcomatous appearance, with spindle cell proliferation. ${ }^{11}$ Whenever disseminated carcinoma is present within the gastrointestinal tract of ar AIDS patient, bleeding may become a problem ${ }^{9,1}$ (Figs. 14-16).

Inflamed, thickened, edematous mucosal folds within the small bowel, which are noticeable or barium examination, are nonspecific findings of infection (Fig. 17). Infection by Giardia lamblia Cryptosporidia, Isospora belli, Strongyloides, anc cytomegalovirus can all have similar radiographic appearances. Hypersecretion from viral or protozoal infection can cause rapid dilution of barium within the small bowel. Discovery of radiographic abnormalities within the small bowel should prompt a thorough microbiologic investigation. At times, endoscopic small bowel biopsy will be required to establish a precise diagnosis.

In contrast to the watery, cholera-like diarrhe resulting from small bowel disease, colitis can re sult in bloody diarrhea teeming with fecal leukocytes. Campylobacter, Shigella, Salmonella, Yer sinia, and Entamoeba histolytica are the most common offending organisms. Cytomegalovirus and Chlamydia trachomatis (lymphogranuloma venereum) can also cause colitis in the AIDS patient. Barium enema reveals a spectrum of disease, 

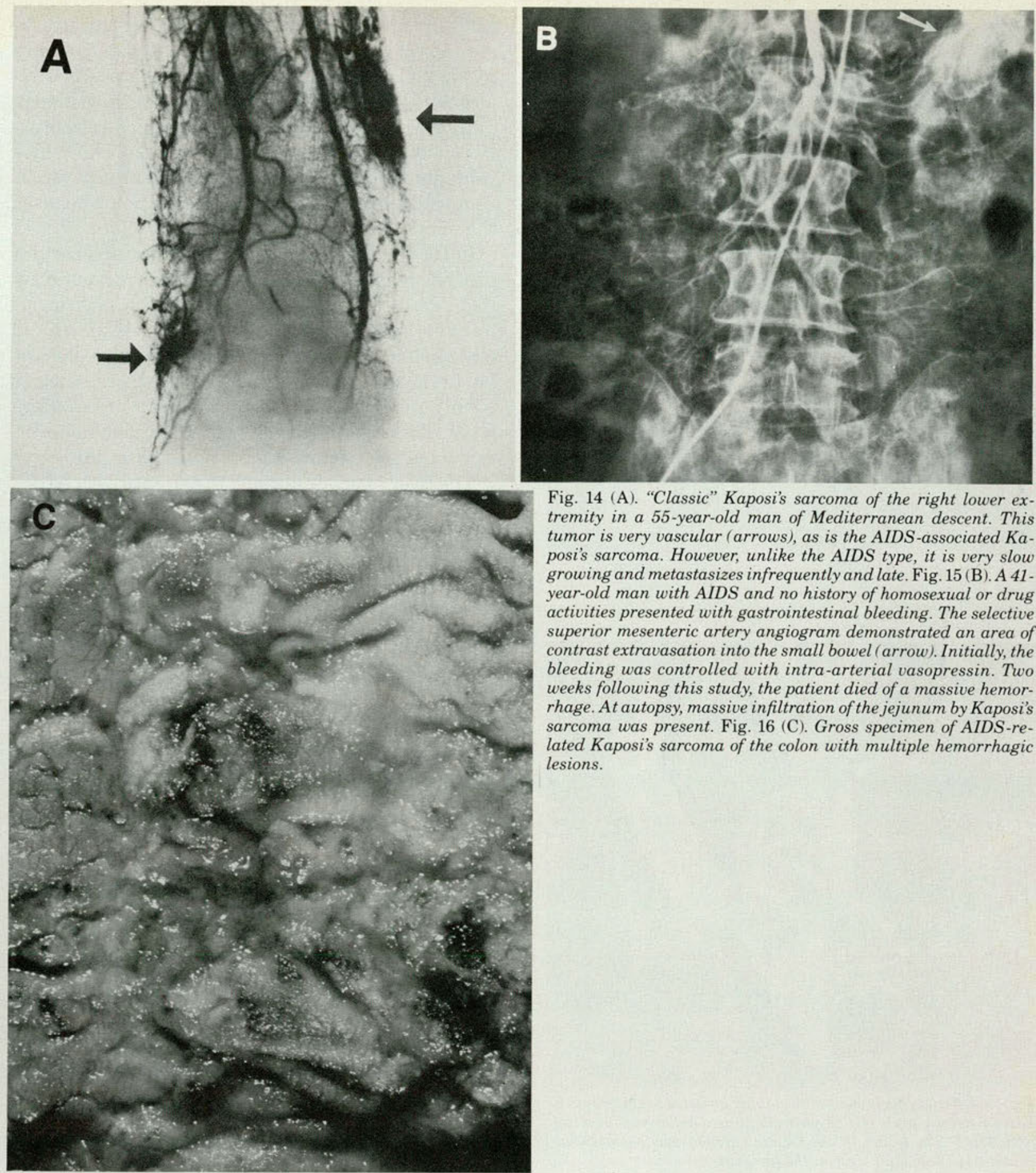

Fig. 14 (A). "Classic" Kaposi's sarcoma of the right lower extremity in a 55-year-old man of Mediterranean descent. This tumor is very vascular (arrows), as is the AIDS-associated Kaposi's sarcoma. However, unlike the AIDS type, it is very slow growing and metastasizes infrequently and late. Fig. 15 (B). A 41 year-old man with AIDS and no history of homosexual or drug activities presented with gastrointestinal bleeding. The selective superior mesenteric artery angiogram demonstrated an area of contrast extravasation into the small bowel (arrow). Initially, the bleeding was controlled with intra-arterial vasopressin. Two weeks following this study, the patient died of a massive hemorrhage. At autopsy, massive infiltration of the jejunum by Kaposi's sarcoma was present. Fig. 16 (C). Gross specimen of AIDS-related Kaposi's sarcoma of the colon with multiple hemorrhagic lesions.

\section{$\mathrm{cm}|\mathrm{IIII}| \mathrm{IIII|} \mid \mathrm{IIII||II|||III||III||}$}




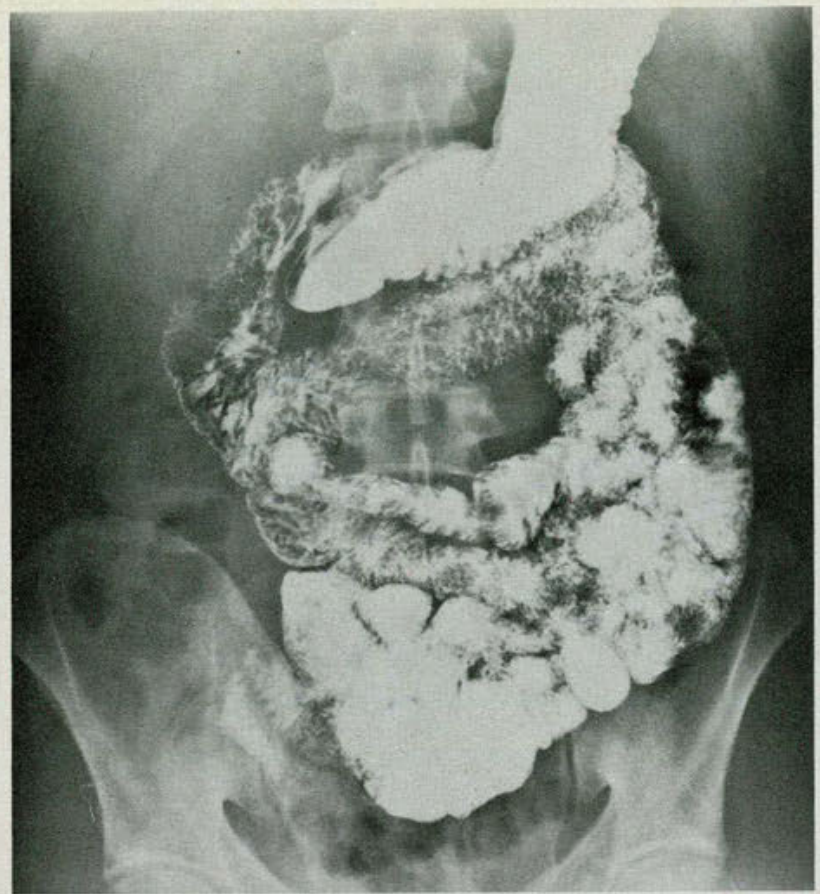

Fig. 17. Small-bowel radiograph from an upper gastrointestinal series in a patient with persistent diarrhea secondary to cryptosporidium infection. The proximal small bowel demonstrates thickening of mucosal folds and nodularity.

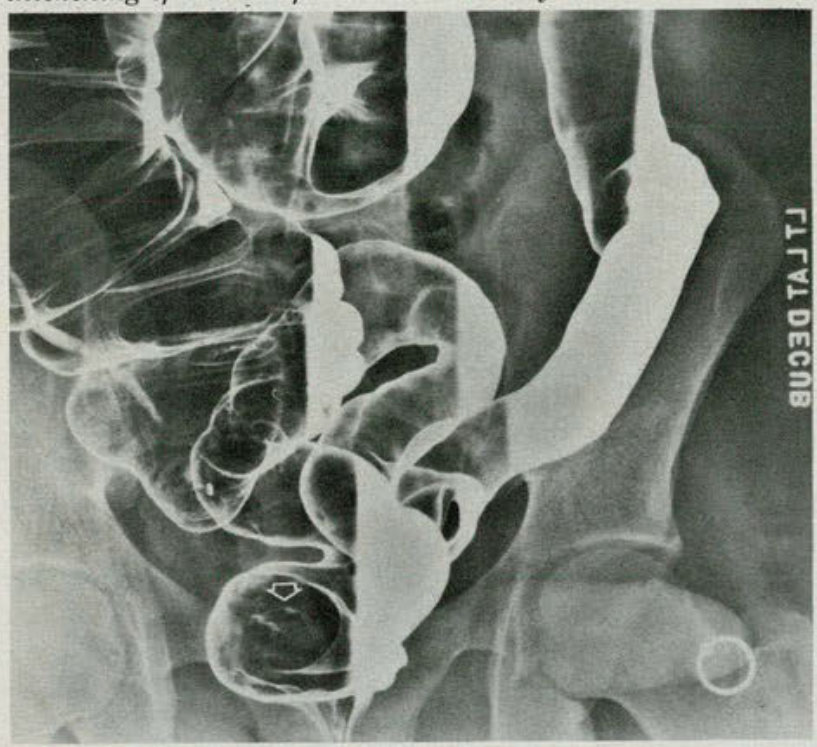

Fig. 18. Left decubitus view of the double-contrast examination in barium enema study of a 28 -year-old patient with rectal bleeding and AIDS. The lesion in the rectum (arrow) was Kaposi's sarcoma. Also note stud ring on the foreskin. ranging from a fine granular mucosal pattern, as seen in early inflammatory bowel disease, to severe ulceration and mucosal sloughing. There is also the possibility that the patient presenting with bloody diarrhea, abdominal pain, and fever has antibioticinduced pseudomembranous colitis. The widespread use of antibiotics in this patient population should arouse suspicion of this condition whenever the symptoms are compatible.

In the homosexual patient, the anus and rectum can be involved by infectious proctitis caused by Neisseria gonorrhoeae, Treponema pallidum, herpes simplex virus, cytomegalovirus, or Chlamydia trachomatis. This area is quite accessible to the flexible sigmoidoscope, so the radiologist is only rarely called upon to make an initial diagnosis. This region is routinely examined, however, as part of the barium enema study (Fig. 18).

The liver may be enlarged in patients with AIDS. Often, a nonspecific fatty change is found on histologic examination. Also seen are sinusoidal dilatation and liver cell atrophy. Surprisingly, acute hepatitis is uncommon. ${ }^{9}$ Pyogenic and fungal abscesses or involvement by malignant disease are more serious causes of hepatic enlargement. Evaluation is best accomplished by CT scan, ultrasonography, or nuclear medicine studies (Figs. 19-21).

\section{Pulmonary manifestations}

More than any other single manifestation of AIDS, pneumocystis pneumonia has become identified with diagnosis of the disease. Pneumocystis carinii, a protozoan, is the most common life-threatening opportunistic infection in this disease; it occurs in 65-80 percent of AIDS patients during the course of their illness. ${ }^{11}$ As many as 50 percent of patients present with pneumocystis pneumonia as the initial manifestation. At first, symptoms may be subtle and consist of subjective shortness of breath and a dry cough. Fever may or may not be present. Early radiographs can be normal. As the pneumonia progresses, a subtle increase in interstitial markings will occur; these may be obvious only by comparison of serial radiographs (Fig. 22). Untreated, the disease will progress to respiratory failure, and at that point the chest radiographs will reveal diffuse interstitial and alveolar pneumonia (Fig. 23). The mortality rate is $30-50$ percent. ${ }^{11}$ Diagnosis usually is made by transbronchial biopsy or bronchoalveolar lavage; open-lung biopsy rarely is necessary. ${ }^{13}$ The use of gallium lung scanning (Fig. 24) has been advocated for early diagnosis of pneumocystis pneumonia when the patient is symptomatic but the chest radiograph is still normal. Although it is quite sensitive, gallium scanning 
suffers from a lack of specificity. The presentation of pneumocystis pneumonia appears similar to that of cytomegalovirus pneumonia, an entity that can also clinically simulate the pneumocystis type, but for which there is as yet no effective treatment.

Atypical mycobacteria, most commonly $M y$ cobacterium avium-intracellulare, presents frequently in the AIDS population. Although typically found in the lungs, this infection is present in an extrapulmonary site in approximately 30 percent of patients. ${ }^{11}$ Mycobacterial tuberculosis, Candida albicans, and Histoplasma capsulatum can present similarly (Figs. 25-27). Coccidioidomycosis can also manifest with the typical grape-skin-walled cavities (Figs. 28 and 29).

Although it has an attack rate three times higher in AIDS patients than in the general population, bacterial pneumonia is not as common in AIDS as one might expect. When present, infection is associated with difficulty in opsonization because of a Bcell defect. Infection by encapsulated bacteria, such as Streptococcus pneumoniae and Hemophilus influenzae are, therefore, most common. ${ }^{14}$ Opportunistic pulmonary infection by Listeria, Salmonella, and Nocardia also occurs.

As in other organ systems, the lungs are sites for metastasis from Kaposi's sarcoma (Fig. 30). On chest radiography, interstitial or alveolar opacification of varying degrees may be demonstrated. Usually, differentiation from opportunistic infection is difficult at best. Cough and dyspnea are universal features of lung metastasis by Kaposi's sarcoma. Fever occurs more often in those patients with concomitant opportunistic infection. Bloody, exudative pleural effusions are a feature of pulmonary Kaposi's sarcoma, whereas they are rare in opportunistic infection. The diagnosis of pulmonary Kaposi's sarcoma is not easy to make by bronchoscopic biopsy. Reliable diagnosis requires histologic examination of a large piece of tissue, which can only be provided by open-lung biopsy. ${ }^{15,16}$

\section{Conclusion}

The AIDS epidemic has led to a devastating clinical problem-an immunocompromised host susceptible to a wide range of opportunistic and malignant diseases. Diagnosis is made difficult by the nonspecificity of the clinical and radiographic findings. The frequency of infection by a number of organisms and the occurrence of simultaneous malignant and infectious processes make definitive diagnosis especially difficult. Tissue biopsy is necessary to direct specific therapy. Interventional procedures, however, are fraught with problems in the AIDS patient because of his/her immunocompromised state. Various radiographic eval-
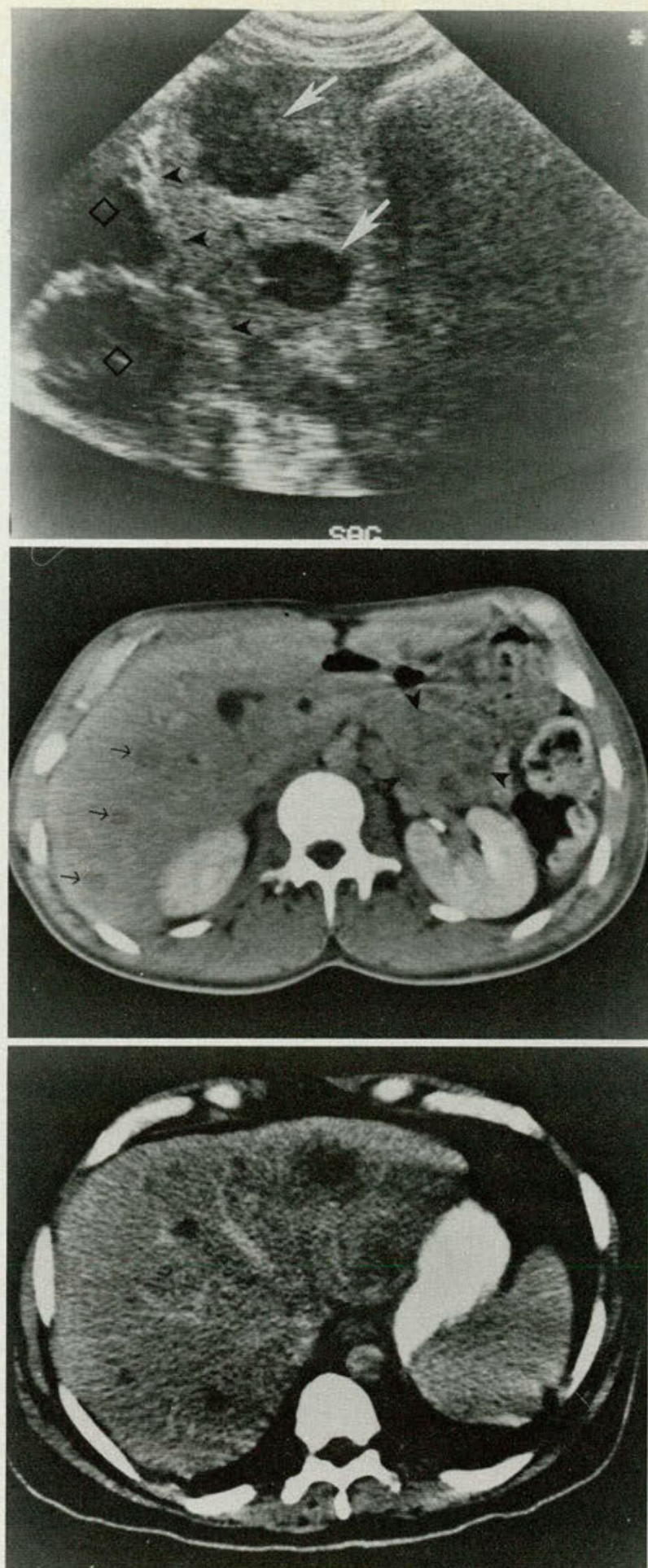

Fig. 19 (top). Sagittal ultrasonographic examination of the left lobe of the liver shows multiple hypoechoic lesions (arrows) secondary to lymphoma. The diaphragm (arrowheads) and heart (diamonds) are viewed superior to the liver. Abscess may give a similar appearance on ultrasonography. Biopsy is often necessary for differentiation. Fig. 20 (center). CT scan of the liver and upper abdomen. Massive retroperitoneal adenopathy (arrowheads) and multiple hepatic lesions (arrows) secondary to nonHodgkins lymphoma are shown. Fig. 21 (bottom). Several lesions of the liver secondary to abscess in an AIDS patient are demonstrated on CT scan. 


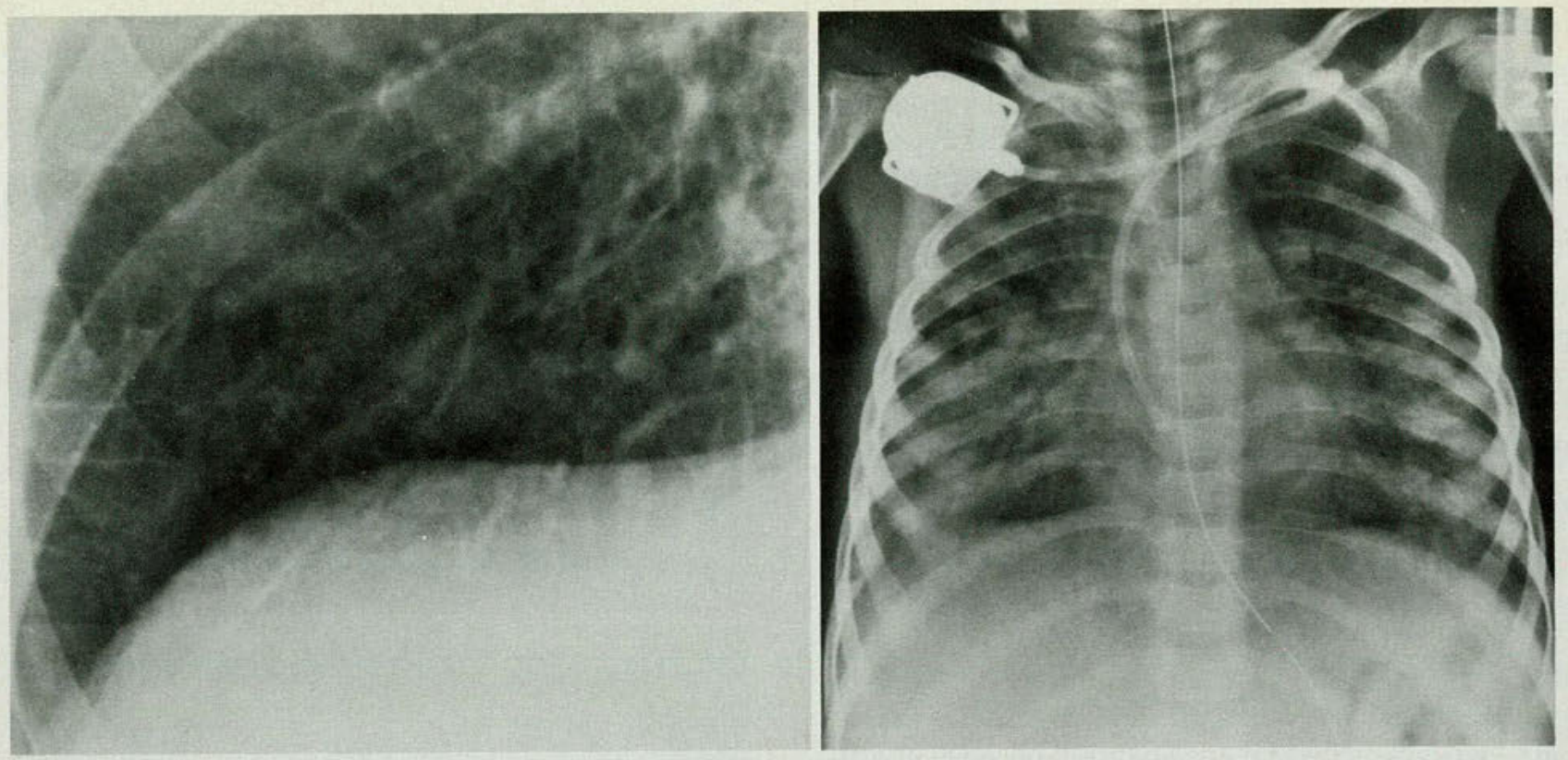

Fig. 22. Findings on a chest $x$-ray may be minimal, such as these Kerley B lines, which are indicative of early interstitial infiltrate from Pneumocystis carinii. Fig. 23. Advanced pneumocystis pneumonia has the classic pattern of perihilar diffuse densities with air bronchograms. This patient, a 3-year-old child, developed transfusion-related AIDS.

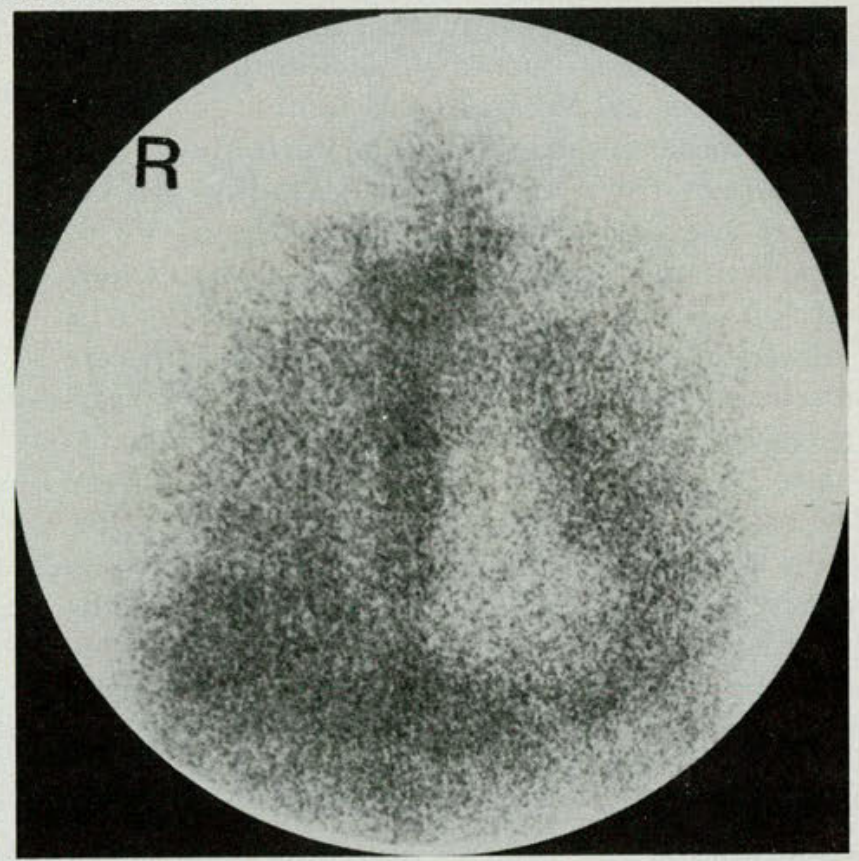

Fig. 24. Gallium 67 scanning may be very helpful when Pneumocystis carinii infection is suspected but the chest $x$-ray is negative. Note the negative heart shadow in this patient, and the diffuse increased uptake in both lung fields. This finding is nonspecific and may be seen in any diffuse process involving the lungs. 
uations are often the first objective demonstration of the disease process. In AIDS, early recognition of disease by appropriate radiographic examination, followed by selective biopsy and specific therapy, might at least decrease the morbidity and mortality of this devastating disease in the short term.

1. Federal Drug Administration: Progress on AIDS. FDA Drug Bull $15: 1-8$, Oct 85

2. Holland, J.C., and Tross, S.: The psychosocial and neuropsychiatric sequelae of the acquired immunodeficiency syndrome and related disorders. Ann Intern Med 103:760-4, Nov 85

3. Marwick, C.: Neurological complications appear often in AIDS. JAMA 253:3379,83, 21 Jun 85

4. Snider, W.D., et al.: Neurological complications of acquired immune deficiency syndrome. Analysis of 50 patients. Ann Neurol 14:403-18, Oct 83

5. Shaw, G.M., et al.: HTLV-III infection in brains of children and adults with AIDS encephalopathy. Science 227:177-82, 11 Jan 85

6. Kelly, W.M., and Brant-Zawadzki, M.: Acquired immunodeficiency syndrome. Neuroradiologic findings. Radiology 149:485-9, Nov 83

7. Whelan, M.A., et al.: Acquired immunodeficiency syndrome. Cerebral computed tomographic manifestations. Radiology 149:477-84, Nov 83

8. Horowitz, S.L., et al: CNS toxoplasmosis in acquired immunodeficiency syndrome. Arch Neurol 40:649-52, Oct 83

9. Welch, K., et al.: Autopsy findings in the acquired immune deficiency syndrome. JAMA 252:1152-9, 7 Sep 84

10. Tavitian, A., Raufman, J-P., and Rosenthal, L.E.: Oral candidiasis as a marker for esophageal candidiasis in the acquired immunodeficiency syndrome. Ann Intern Med 104:54-5, Jan 86
11. Fauci, A.S., et al:: The acquired immunodeficiency syndrome. A update. Ann Intern Med 102:800-13, Jun 85

12. Lind, S.E., et al.: Malignant lymphoma presenting as Kaposi's sar coma in a homosexual man with the acquired immunodeficiency syn drome. Ann Intern Med 102:338-40, Mar 85

13. Broaddus, C., et al.: Bronchoalveolar lavage and transbronchial bi opsy for the diagnosis of pulmonary infections in the acquired immu nodeficiency syndrome. Ann Intern Med 102:747-52, Jun 85

14. Polsky, B., et al.: Bacterial pneumonia in patients with the acquire immunodeficiency syndrome. Ann Intern Med 104:38-41, Jan 86

15. Murray, J.F., et al.: Pulmonary complications of the acquired immu nodeficiency syndrome. Report of a National Heart, Lung, and Bloo Institute workshop. N Engl J Med 310:1682-8, 21 Jun 84

16. Ognibene, F.P., et al.: Kaposi's sarcoma causing pulmonary infiltrate and respiratory failure in the acquired immune deficiency syndrome Ann Intern Med 102:471-5, Apr 85

Accepted for publication in November 1986.

At the time this paper was written, Dr. Pera was a fellow ir diagnostic radiology at the University of Texas M.D. Andersor Hospital and Tumor Institute. He is now an attending radi ologist at the Michigan State University, College of Osteopathi Medicine, East Lansing, Michigan. He is certified in family practice and radiology. Dr. Weisgrau is a resident in radiology at the M.D. Anderson Hospital and Tumor Institute, where Dr Harle is an attending radiologist, and Dr. Rios is an attending physician in the Department of Hematology and Oncology.

Dr. Pera, Michigan State University, College of Osteopathi Medicine, Department of Radiology, B-220 Clinical Center, Eas Lansing, Michigan 48824. 